\title{
Cases of abdominal wall endometriosis distinguished from soft tissue tumors: A report of three cases
}

\author{
TOMOHITO HAGI, TOMOKI NAKAMURA, TAKAHIRO HASEGAWA, KUNIHIRO ASANUMA and AKIHIRO SUDO
}

Department of Orthopaedic Surgery, Mie University Graduate School of Medicine, Tsu-city, Mie 5148507, Japan

Received June 8, 2020; Accepted August 27, 2020

DOI: $10.3892 /$ br.2020.1371

\begin{abstract}
Endometriosis is an estrogen-dependent inflammatory disease characterized by the presence of endometrium-like tissue in sites outside the uterine cavity. It affects $6-10 \%$ of women of reproductive age. Concerning abdominal wall endometriosis, it is particularly rare with a reported incidence of 0.03-3.5\%. Abdominal wall endometriosis may be misdiagnosed as soft tissue tumors. Patients are often referred to an orthopedic department, although this is not familiar to orthopedic surgeons. In the present report, we describe three women with abdominal painful mass who had previously undergone Caesarean section. The masses were associated with their menstrual cycle and existed proximal to the Caesarean section surgical scar. Pelvic magnetic resonance imaging revealed isointense or hyperintense to muscle on both T1- and T2-weighted images. All patients were suspected of suffering from abdominal wall endometriosis, and were treated with complete surgical resection and developed no recurrence. The pathological findings confirmed endometrial gland and endometrial stroma features. Clinical symptoms and medical history play an important role in the diagnosis of abdominal wall endometriosis in addition to radiological examinations.
\end{abstract}

\section{Introduction}

Endometriosis is a benign tumor, classically defined as the presence of endometrial tissues at sites outside the uterine cavity. Endometriosis affects $6-10 \%$ of women of reproductive age (1). The most common symptoms of endometriosis include dysmenorrhea, dyspareunia, chronic pelvic pain, and infertility (2). Abdominal wall endometriosis arises in the location above the peritoneum or in scar tissues. Abdominal wall endometriosis is rare, with a reported incidence of $0.03-3.5 \%$ (3). Although Caesarean section surgical scars are

Correspondence to: Dr Tomoki Nakamura, Department of Orthopaedic Surgery, Mie University Graduate School of Medicine, 2-174 Edobashi, Tsu-city, Mie 5148507, Japan

E-mail: tomoki66@clin.medic.mie-u.ac.jp

Key words: abdominal wall, endometriosis, soft tissue tumors, caesarean section, case report the most common sites of abdominal wall endometriosis, it has also been reported in laparoscopic trocar tracts or at episiotomy incision sites (4). It is often misdiagnosed as a hernia, hematoma, abscess, or lipoma because of its rarity (5-7). The high rate of misdiagnosis causes unnecessary procedures with increased emotional and physical distress among these patients (8). It is not uncommon to refer patients to an orthopedic department since physicians suspect that a mass may be an abdominal wall tumor, but this is not familiar to orthopedic surgeons. Herein, we describe three cases of abdominal wall endometriosis. Written informed consent for the present report was obtained from all patients. Institutional review board approval was waived because of the nature of this study.

\section{Case reports}

Case 1. A 44-year-old healthy woman developed a lower abdominal painful mass 3 years prior. She mentioned that the mass was associated with her menstrual cycle. She had undergone Caesarean section 6 years before the initial presentation. At the clinical examination, a painful, elastic, hard, round mass approximately $30 \mathrm{~mm}$ in diameter was observed above her Caesarean section surgical scar. Pelvic magnetic resonance imaging (MRI) revealed a 25-mm mass in the subcutaneous abdominal wall. The mass showed isointensity compared to the muscle on both T1- and T2-weighted images (Fig. 1). We suspected abdominal wall endometriosis because of the patient's medical history, clinical symptoms, and radiological examination findings. We planned to resect the tumor to confirm the diagnosis. The pathological findings confirmed endometrial gland and endometrial stroma features; the final diagnosis was endometriosis. Adequate surgical margins were acquired at a distance of $10 \mathrm{~mm}$. At the 3-year follow-up, there was no recurrence, and she did not experience pain.

Case 2. A 37-year-old woman developed a lower abdominal painful mass 3 years prior. She mentioned that the mass was associated with her menstrual cycle. She had undergone Caesarean section twice: 5 and 7 years before the initial presentation. At the clinical examination, a painful, elastic, hard, round mass approximately $20 \mathrm{~mm}$ in diameter was detected $20 \mathrm{~mm}$ proximal to the Caesarean section surgical scar. Pelvic MRI revealed a $15-\mathrm{mm}$ mass in the subcutaneous abdominal wall. The mass showed hyperintensity compared to the muscle on both T1- and T2-weighted images (Fig. 2). As 

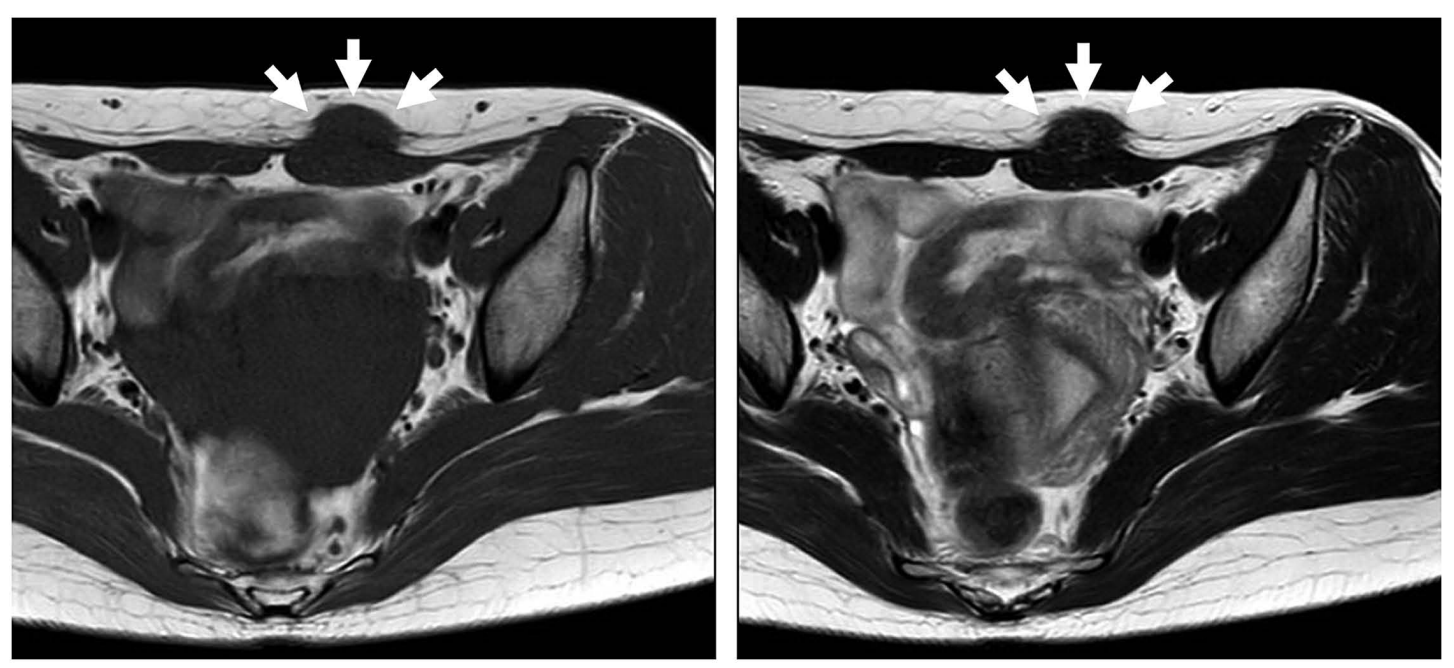

Figure 1. Unenhanced axial T1-weighted MRI (left), T2-weighted MRI (right) showing nodule isointensity to muscle on both T1- and T2-weighted MRI in the subcutaneous abdominal wall. MRI, magnetic resonance imaging (arrow).
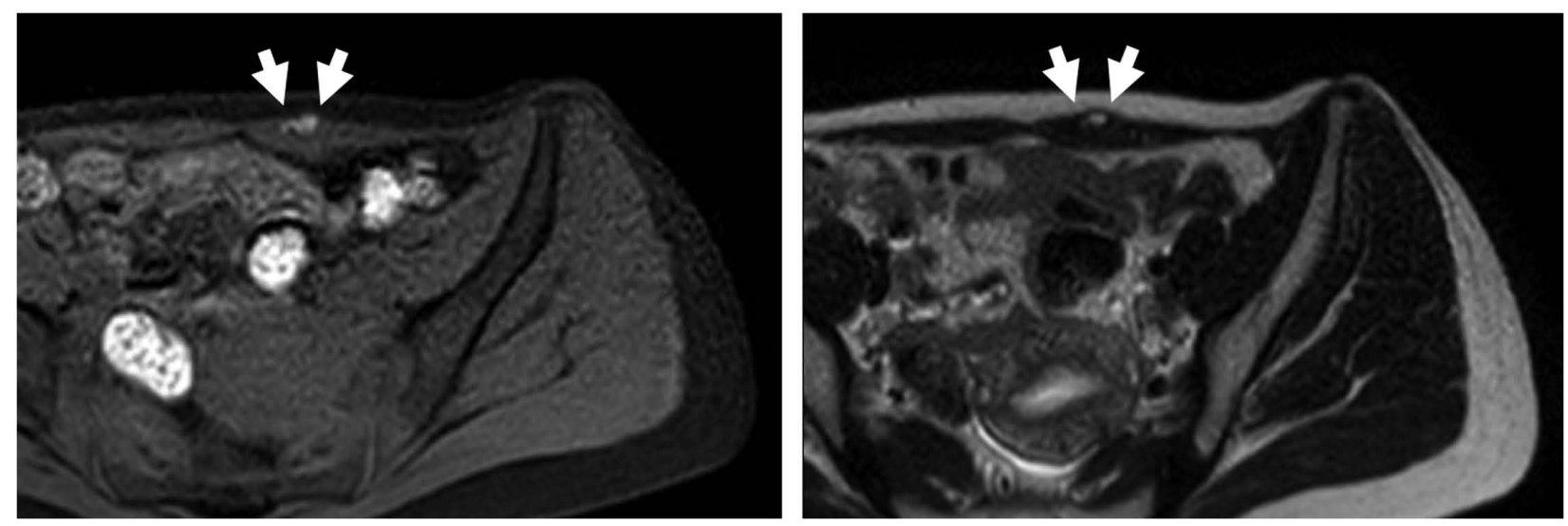

Figure 2. Unenhanced axial T1-weighted MRI (left), T2-weighted MRI (right) showing nodule hyperintensity to muscle on both T1- and T2-weighted MRI in the subcutaneous abdominal wall. MRI, magnetic resonance imaging (arrow).
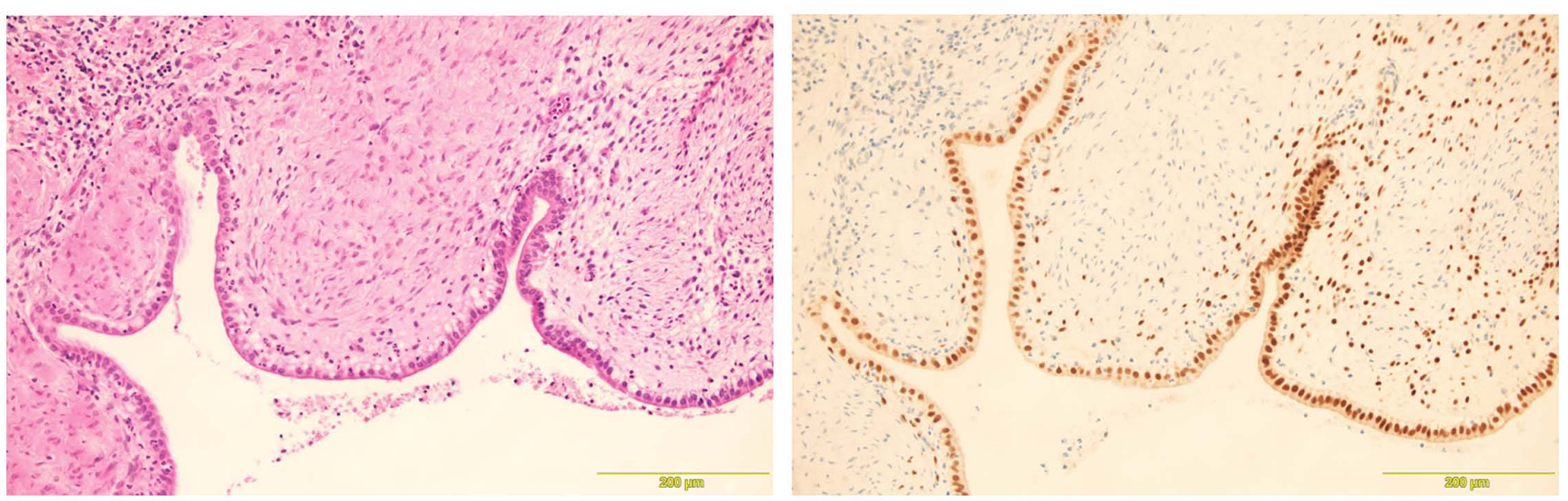

Figure 3. Histological analysis of the lesion with hematoxylin and eosin staining showing both endometrial gland and endometrial stroma features (left), and estrogen receptor positivity on immunohistochemical staining (right).

we suspected abdominal wall endometriosis, tumor resection was performed. Estrogen receptors tested positive on immunohistochemical analysis (Fig. 3). At the 1-year follow-up, there was no recurrence, and she did not experience pain.
Case 3. A 26-year-old woman developed a lower abdominal painful mass 6 months prior. She had undergone Caesarean section twice: 1 and 5 years before the initial presentation. At the clinical examination, a painful, elastic, hard, round mass 

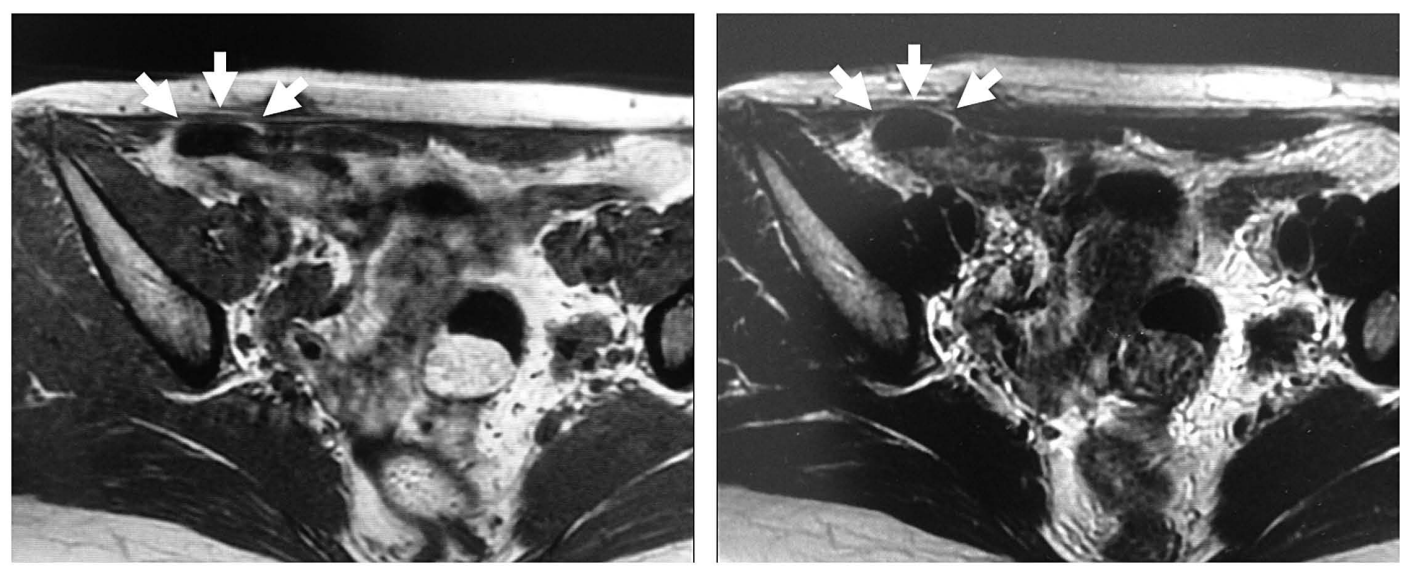

Figure 4. Unenhanced axial T1-weighted MRI (left), T2-weighted MRI (right) showing nodule isointensity to muscle on both T1- and T2-weighted MRI in the subcutaneous abdominal wall. MRI, magnetic resonance imaging (arrow).

approximately $30 \mathrm{~mm}$ in diameter was detected $5 \mathrm{~mm}$ to the right of her Caesarean section surgical scar. Pelvic MRI revealed a $20-\mathrm{mm}$ mass in the subcutaneous abdominal wall. The mass showed isointensity compared to the muscle on both T1- and T2-weighted images (Fig. 4). As we suspected abdominal wall endometriosis, tumor resection was performed. At the 1-year follow-up, there was no recurrence, and she did not experience pain.

\section{Discussion}

The most evident clinical manifestation of endometriosis is a painful subcutaneous mass with cyclic pain associated with menses (9). The cyclical nature of the complaint is present in $40-73 \%$ of cases $(7,8,10)$. The symptoms are often associated with a history of development of a palpable mass within or adjacent to a surgical scar. The incidence of endometriosis after a Caesarean section has been estimated to be $0.26 \%$ in a 25-year interval (3). Another paper published by Singh et al reported a similar incidence, i.e., $0.2 \%$ in a 10 -year interval (11). The average duration between Caesarean section surgery and the onset of symptoms is 3.7-4.5 years (12). The incidence of endometriosis after a Caesarean section shows a significantly high relative risk of 3.3 compared to that of endometriosis associated with scars concerning other surgical procedures, such as laparoscopic trocar or episiotomy (3). This high risk can be explained by the higher exposure of endometrial cells to the subcutaneous tissue during the procedure. Some studies have demonstrated the use of oral contraceptives, gonadotropin-releasing hormone analogs, danazol, and progesterone, which are approved for treating pelvic endometriosis (13). However, complete surgical excision of endometriotic lesions is required to avoid recurrence. The recurrence rate with inadequate resection has been reported as $4.5-9.1 \%$ (8). In the present cases, adequate surgical margins were acquired and there were no recurrences.

The pathogenesis of endometriosis is explained mainly by the metaplasia theory, embryonic rest theory, or transport theory. The metaplasia theory suggests that primitive mesenchymal cells undergo specialized differentiation of metaplasia to form endometrial implants (11). The embryonic rest theory hypothesizes that Müllerian remnants can differentiate into endometrial tissue, which may cause symptoms (12). The transport theory, specifically the implantation theory, suggests that endometrial cells escape through an incision made in the uterus during the surgical procedure and are implanted within the abdominal wall (14). In our study, all patients were young women of reproductive age, had a history of prior Caesarean section, and harbored an abdominal mass in or adjacent to their Caesarian surgical scars. These findings suggest a surgically induced iatrogenic implantation etiology.

The use of MRI, computed tomography, ultrasonography, and fine-needle aspiration in clinical practice for the diagnosis of abdominal wall endometriosis is well reported $(15,16)$. Although these diagnostic techniques can adequately depict abdominal wall lesions, these imaging modalities cannot provide a definitive preoperative diagnosis. Concerning MRI, typical ovarian endometriosis shows hyperintensity on T1-weighted images and hypointensity on T2-weighted images. On the other hand, in the abdominal wall, endometriosis may be isointense or mildly hyperintense to muscle on both T1- and T2-weighted images. In some cases, there may be cystic areas of hemorrhage within abdominal wall endometriosis that appear homogeneously hyperintense on T1-weighted images $(13,17)$. Among the present cases, two patients showed hyperintensity to the muscle on both T1- and T2-weighted images, and one patient showed isointensity on both T1- and T2-weighted images. Therefore, hematoma, suture granuloma, or desmoid may be considered among the differential diagnoses of abdominal wall endometriosis. The reported accurate preoperative diagnosis rate varies between 20 and $50 \%(6,10)$. We decided to perform wide resection in all cases since function could be preserved. A possible explanation of diagnostic failure is that the diagnoses are almost always confirmed by physicians who are not very familiar with this entity. Therefore, we must obtain the needed patient medical histories and perform physical examinations. Finally, we can be led to suspect abdominal wall endometriosis based on several findings including medical history, typical symptoms, and radiological examinations.

\section{Acknowledgements}

Not applicable. 


\section{Funding}

No funding was received.

\section{Availability of data and materials}

Additional information concerning the three case reports is available from the corresponding author upon reasonable request.

\section{Authors' contributions}

TN conceived the study, treated the patients, collected the data and wrote the manuscript. THag collected, analyzed and interpreted the clinical data. THas and KA performed the surgery, analyzed and interpreted the clinical data. AS analyzed and interpreted the clinical data and reviewed the manuscript. All authors read and approved the manuscript and agree to be accountable for all aspects of the research in ensuring that the accuracy or integrity of any part of the work are appropriately investigated and resolved.

\section{Ethics approval and consent to participate}

Written informed consent for the present report was obtained from all patients. Institutional review board approval was waived because of the nature of this study.

\section{Patient consent for publication}

Written informed consent was obtained from the patients for the publication of the case details and associated images.

\section{Competing interests}

The authors declare that they have no competing interests.

\section{Authors' information}

Tomohito Hagi: ORCID: 0000-0002-2937-3447.

\section{References}

1. Burney RO and Giudice LC: Pathogenesis and pathophysiology of endometriosis. Fertil Steril 98: 511-519, 2012.
2. Cozzolino M, Magnolfi S, Corioni S, Moncini D and Mattei A: Abdominal wall endometriosis on the right port site after laparoscopy: Case report and literature review. Ochsner J 15: 262-264, 2015.

3. Nominato NS, Prates LF, Lauar I, Morais J, Maia L and Geber S: Caesarean section greatly increases risk of scar endometriosis. Eur J Obstet Gynecol Reprod Biol 152: 83-85, 2010.

4. Gidwaney R, Badler RL, Yam BL, Hines JJ, Alexeeva V, Donovan V and Katz DS: Endometriosis of abdominal and pelvic wall scars: Multimodality imaging findings, pathologic correlation, and radiologic mimics. Radiographics 32: 2031-2043, 2012.

5. Tazaki T, Oue N, Ichikawa T, Tsumura H, Hino H, Yamaoka H, Kanehiro T and Yasui W: A case of endometriosis of the appendix. Hiroshima J Med Sci 59: 39-42, 2010.

6. Horton JD, Dezee KJ, Ahnfeldt EP and Wagner M: Abdominal wall endometriosis: A surgeon's perspective and review of 445 cases. Am J Surg 196: 207-212, 2008.

7. Ozel L, Sagiroglu J, Unal A, Unal E, Gunes P, Baskent E, Aka N, Titiz MI and Tufekci EC: Abdominal wall endometriosis in the cesarean section surgical scar: A potential diagnostic pitfall. J Obstet Gynaecol Res 38: 526-530, 2012.

8. Khan Z, Zanfagnin V, El-Nashar SA, Famuyide AO, Daftary GS and Hopkins MR: Risk factors, clinical presentation, and outcomes for abdominal wall endometriosis. J Minim Invasive Gynecol 24: 478-484, 2017.

9. Woodward PJ, Sohaey R and Mezzetti TP Jr: Endometriosis: Radiologic-pathologic correlation. Radiographics 21: 193-216, 2001.

10. Bektas H, Bilsel Y, Sari YS, Ersöz F, Koc O, Deniz M, Boran B and Huq GE: Abdominal wall endometrioma; a 10-year experience and brief review of the literature. J Surg Res 164: e77-e81, 2010.

11. Singh KK, Lessells AM, Adeam DJ, Jordan C, Miles WF, Macintyre IM and Greig JD: Presentation of endometriosis to general surgeons: A 10-year experience. Br J Surg 82: 1349-1351, 1995.

12. Agarwal $\mathrm{N}$ and Subramanian A: Endometriosis-morphology, clinical presentations and molecular pathology. J Lab Physicians 2: $1-9,2010$.

13. Hensen JH, Van Breda Vriesman AC and Puylaert JB: Abdominal wall endometriosis: Clinical presentation and imaging features with emphasis on sonography. AJR Am J Roentgenol 186: 616-620, 2006

14. Blanco RG, Parithivel VS, Shah AK, Gumbs MA, Schein M and Gerst PH: Abdominal wall endometriomas. Am J Surg 185: 596-598, 2003.

15. Francica G: Reliable clinical and sonographic findings in the diagnosis of abdominal wall endometriosis near cesarean section scar. World J Radiol 4: 135-140, 2012.

16. Stein L, Elsayes KM and Wagner-Bartak N: Subcutaneous abdominal wall masses: Radiological reasoning. AJR Am J Roentgenol 198: W146-W151, 2012.

17. Busard MP, Mijatovie V, van Kuijk C, Hompes PG and van Waesberghe JH: Appearance of abdominal wall endometriosis on MR imaging. Eur Radiol 20: 1267-1276, 2010. 